\title{
LA PARTICIPACIÓN DE LOS ACTORES EN LA INSTITUCIÓN ESCOLAR: UN INSTRUMENTO AL SERVICIO DE LA EMANCIPACIÓN SOCIAL ${ }^{1}$
}

\section{Dra. Michèle Garant*}

Resumen: La autora presente un análisis crítico relativo a la eficacia de la participación en las instituciones, y una interrogación sobre las condiciones de realización y de duración de dicha participación.

El propósito se desarrolla en cinco puntos: (1) los costos y las resistencias que puede suscitar la participación, (2) la participación, un instrumento para la acción en relación con los objetivos como con el proceso, (3) el rol de la participación en las regulaciones sociales y educativas de hoy en día, (4) las condiciones para una participación eficaz y (5) la toma en cuenta de tensiones en las instituciones "que aprenden $\mathbf{t}$

En conclusión, insiste en el trabajo de coherencia necesaria al seno de las instituciones, y en rol importante del liderazgo a todas las etapas de las acciones participativas.

\begin{abstract}
The author presents a critical analysis of participation effectiveness in the institutions, and an interrogation about the conditions of participation achievement and duration.

She develops five points (1) Costs and resistances linked to participations (2) Participation as an acting tool in connection with management objectives and with management processes (3) The role of participation in the social and educational regulations to-day (4) Conditions for an efficient participacion (5) Dilemnas to be considered in learning organizations.

As a conclusion she emphasizes the necessary consistency to be build inside the institutions, and the important role of leadership in supporting every step of the participative action.
\end{abstract}

Palabras clave: Participación/ Emancipación social/ Cooperación conflictual

\section{Introducción}

La comunión de convicciones relativas a la capacidad de las personas de construir en conjunto sus vidas y sus proyectos no permite, de ninguna manera, de acuerdo a nuestra visión, prescindir de un análisis crítico relativo a la eficacia de la participación en las instituciones, ni una interrogación rigurosa sobre las condiciones de realización y de duración de dicha participación. Es por ello que, en este documento, no realizaremos una "arenga ideológica acerca de la participación", más bien intentaremos precisar su eficacia y su pertinencia en el seno de una institución que se considera democrática y equitativa.

\footnotetext{
* Licenciada y Doctora en Psicología de la Universidad de Louvain. Licenciada en Ciencias Pedagógicas de la Universidad de Louvain. Actualmente es profesora de Le Grain: Movimiento de Acción Pedagógica, FUCaM: Facultés Universitaires Catholiques de Mons, Belgique. UCL: Université Catholique de Louvain-la-Neuve, Belgique. e-mail: garant@fucam.ac.be
}

Artículo recibido: 9 de setiembre, 2003

Aprobado: 24 de noviembre, 2003

\footnotetext{
${ }^{1}$ Una versión anterior de este articulo fue publicado en libro La pédagogie émancipatrice, Peter Lang, 2002.

${ }^{2}$ Traducción de « learning organization » used by Peter Senge in The Fifth Discipline, Doubleday, 1990.
} 
En el marco de este escrito, no pretenderemos realizar un trabajo exhaustivo acerca de este tema. Ello requeriría, para ser desarrollado integralmente, la instalación y la puesta en funcionamiento de dispositivos teóricos sistemáticos de comparación y de evaluación de sus resultados. Los agentes en terreno, sean cuales sean sus competencias y su compromiso, generalmente no disponen de tiempo ni de distancia necesaria para realizar dicho trabajo. Por otra parte, los investigadores de nuestro país, generalmente tienen otras prioridades y disponen de pocos recursos para desarrollar este tipo de análisis.

Gracias a nuestra práctica docente de enseñanza y acompañamiento de memorias universitarias realizadas por adultos, disponemos de resultados de algunas investigaciones relativas a este tema. Por otra parte, nuestra implicación personal en movimientos de acción social nos permite, igualmente, realizar algunas observaciones y plantear algunas preguntas, con las precauciones necesarias cuando se es actor implicado.

Desarrollaremos nuestro propósito en cinco puntos: (1) La participación no es mágica: los costos de ella y las resistencias que puede suscitar, (2) la participación, un instrumento para la acción en relación con el pilotaje como con el proceso, (3) lugar de la participación en las regulaciones sociales y educativas de hoy en día, (4) condiciones para una participación eficaz y (5) planteamiento de tensiones para las instituciones "que aprenden"

\section{La participación no es mágica}

\subsection{La toma en consideración de las contingencias en la participación.}

Incluso si el tema de la participación es, hoy en día parte, de la retórica del cambio y de la democracia, nos parece importante recordar que la participación no es automáticamente un "regalo de confianza", realizado en un registro de efusión que garantizaría automáticamente "el" éxito en el "mejor de los mundos".

Numerosos estudios de psicología social y de análisis de las organizaciones han demostrado ampliamente que una decisión colectiva no es necesariamente más eficaz que una decisión individual, y que los productos de la institución no son necesariamente mejorados, ni cuantitativamente ni cualitativamente, por la existencia de dispositivos de participación.

\footnotetext{
${ }^{3}$ Traducción de « learning organization » used by Peter Senge in The Fifth Discipline, Doubleday, 1990.
} 
Así también, como lo han desarrollado los teóricos de la contingencia, no se participa "para participar". Si se participa es en el marco de una actividad y de circunstancias precisas; y el tipo de producción o de servicio, las condiciones de contexto, la historia y la cultura de la institución, las competencias y las convicciones de los actores y la calidad del líder, modifican la eficacia de un dispositivo de participación en la institución.

Nuestro modelo de análisis no toma en consideración únicamente las condiciones de contexto. Nuestra modelización busca tomar en cuenta, por una parte, el juego de influencia de los actores, los sistemas de acción que ellos constituyen, y, por otra parte, los valores a los que ellos se refieren y la acción que construyen en una voluntad de cambio social.

\subsection{Costos de la participación}

El desarrollo de la participación no es una inversión de "suma nula". La implantación, la evaluación y el mantenimiento de dispositivos de participación son costosos desde diversos puntos de vista, si se comparan, por ejemplo, con la eficacia (en el corto plazo al menos) de las estandarizaciones burocráticas o de la Organización Científica del Trabajo, la OST de F. Taylor.

Para aquellos que no se hubiesen dado cuenta, aquí se detallan algunos de los costos posibles de la instalación y puesta en marcha de dispositivos de participaciónt

- costos energéticos: cambio de ritmos de aprendizaje, aculturación,

- costos físicos: abandono de rutinas (de obediencia o de rebelión) o de hábitos de dirección,

- costos emocionales: estrés a causa de la responsabilidad, el control del grupo, en la necesidad de soporte,

- costos relacionales: toma en cuenta de elementos en juego y de los conflictos que están relacionados a ellos y a las opiniones diferentes

- costos de dependencia relacionada a los compromisos,

- costos económicos relacionados a los tiempos, lugares, competencias a prever o modificar.

\footnotetext{
${ }^{4}$ Philippe Hermel, Le management participatif, París: Les éditions d'organisation, 1988.
} 
Un interlocutor puede encontrar este cálculo poco generoso. No obstante, un cierto pragmatismo me parece necesario puesto que, ya en la segunda mitad de la vida profesional bien avanzada yo he observado (sin perder por ello todas mis convicciones), en numerosas ocasiones, cómo un compromiso total y coherente de ciertos actores no los prevé, en ningún caso, de un derrumbe o de un cambio de rumbo pasadas una o dos decenas de años. Ello bien merece, me parece, una atención particular hacia la regulación y la revitalización de los recursos, tanto desde el punto de vista de las personas como desde el punto de vista de la gestión global de las energías en la institución.

\subsection{Resistencias suscitadas por la participación}

La participación puede suscitar resistencia. No obstante, y siguiendo a M. Crozier negamos a considerar la "resistencia al cambio" como una etapa obligatoria del desarrollo de éste, o incluso como un fenómeno "natural" en la vida de todo grupo. Las resistencias son reacciones de los actores construidas en relación con las culturas, los grupos de pertenencia, los temores de perder lo que les constituye, los duelos de dejar los hábitos, las certezas, las seguridades. Ellas deben también ser analizadas, por respeto a los interlocutores como para permitir las estrategias de desarrollo de dispositivos más participativos.

Estas resistencias pueden ser cognitivas (falta de información, desconocimiento de los lenguajes utilizados, desconocimiento del contenido de aquello que es promovido), afectivas (temor de la dificultad, de la falta de eficacia, miedo a la diferencia, rechazo de la decisión colectiva), ideológicas (oposición a la cooperación, confianza en la jerarquía, en los expertos, en los elegidos), ergonómicas (perjuicios o aumento de cargas físicas o mentales percibidas como negativas)

\section{La participación, un instrumento para la acciónd}

\subsection{Características de la participación}

(1) Una "gramática" de la participación

El término "participación" puede ser utilizado en distintos niveles lógicos y en el marco de registros de valor bien diferentes.

\footnotetext{
${ }^{5}$ Michel Crozier, L'acteur et le système, Paris: Seuil, 1977.

${ }^{6}$ Daniel J. Mothe-Gautrat, Méthode d'évaluation des systèmes participatifs, París: CNRS, 1984.

${ }^{7}$ En esta parte, así como en la parte número 4, nos inspiramos en Ph. Hermel, op. cit.
} 
Retomemos a Herme $\mathbf{0}$ (ver en la siguiente página). El esquema en el cual él presenta diferentes formas de la participación: Se trata ¿de una participación en los medios, en los resultados, en los procesos?, y en el último caso, se participa ¿en la concepción, en la realización o en la ejecución de una actividad?

Complicando el problema aún más todavía, se trata de ¿participar al nivel de microsistemas, en tanto miembro de un grupo o de un equipo, o bien al nivel de un mesosistema como por ejemplo un servicio o de la institución, o incluso al nivel de macrosistema como una región o un país?

Esta gramática de la participación no da indicación alguna sobre el espíritu (o ideología) que preside la utilización de este instrumento. La intención de aquel que promueve la participación no es necesariamente emancipadora. Siguiendo los propósitos de L. Peeters en su reciente obra [ así como estas intenciones pueden ser democráticas, ellas bien pueden ser comunitarias, de adaptación, utópicas, terapéuticas, formativas, administrativas o... demagógicas.

De seguro, sería muy interesante estudiar hasta qué punto las miradas democráticas conducen a efectos emancipadores, así como saber si otros registros distintos del democrático pueden, también, tener efectos emancipadores.

${ }^{8}$ Hermel, op. cit., p. 5.

${ }^{9}$ Luc Peeters, La concertation dans l'enseignement, Bruselas: De Boeck, 1991, Ch. VI. 


\section{Las diferentes naturalezas de la participación}

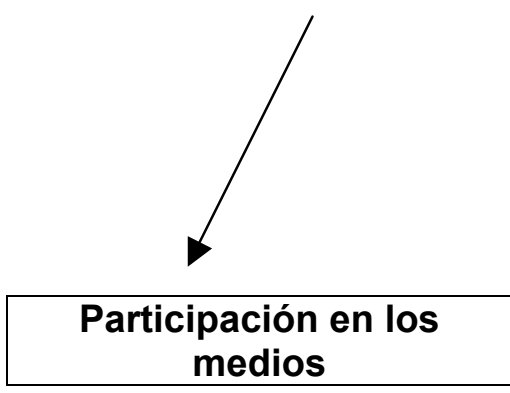

(Entrantes)

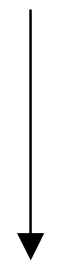

Aporte

(Agregación de riqueza)

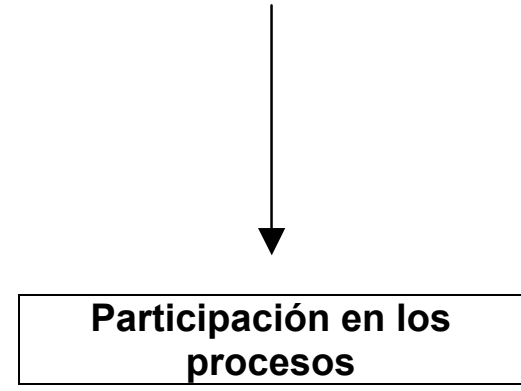

(Creación de valor agregado)

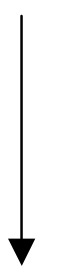

Acción

(Creación de riqueza)

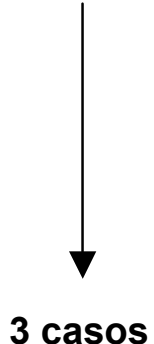

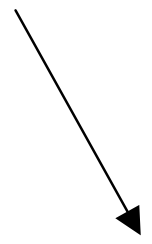

Participación en los resultados

(Salientes)

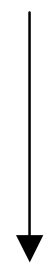

Recepción

(Repartición de riqueza)

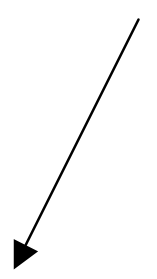

Participación de la concepción
Participación de

la realización

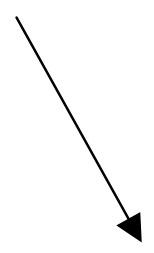

Participación en la ejecución

(2) Definición de una participación democrática 
Presentamos más abajo una definición que aplica una acepción del término que se utiliza en las sociedades o en las instituciones democráticas que apuntan al desarrollo de la autonomía de los ciudadanos. La promoción de la participación en este espíritu supone la adhesión a valores relativos:

- a la autonomía de las personas y a su educabilidad ("todos capaces");

- al desarrollo de un proyecto común, de un espíritu de equipo ("tener éxito en conjunto");

- a la utilización de herramientas privilegiadas como la negociación, la delegación, el compartir.

La participación busca desarrollar en el participante un comportamiento responsable y “concebidor". La administración y la gestión participativas de una institución o de un proyecto busca la autonomía formal de los participantes, en coherencia con las finalidades de la organización, y da a cada uno las condiciones de reaccionar con un cierto grado de autonomía que conduce a preparar (por ejemplo, gracias al análisis del contexto, de las prioridades), a tomar (por ejemplo, gracias al análisis de los recursos), a poner en marcha (por ejemplo, a través del análisis del proceso) o a evaluar (por ejemplo, a través del análisis del cambio producido) decisiones y acciones de manera tal que las permita mejorar.

Encontramos aquí cinco características:

- un compartir la decisión y la actividad, tomando en cuenta la necesidad de información y de debate;

- una gestión global y multidimensional, incluso en actividades aparentemente menores;

- una articulación entre el individuo, el grupo y la organización;

- la búsqueda de sinergias;

- una complementaridad entre el funcionamiento cotidiano y la toma de distancia gracias al análisis y la evaluación, una conjugación entre el corto y mediano plazo, y también un trabajo de memoria.

Una cultura de participación implica que los individuos sean considerados en su totalidad, como seres dotados de facultades de creación, capaces de tener éxito en tareas de acuerdo 
a sus niveles, teniendo una posibilidad de gestión de sus vidas, preocupados de cooperar, y por ende, "inteligentes de ellos mismos", según la fórmula utilizada por Ph. Meirieu四

Por otra parte, en las instituciones sociales y educativas actuales, se trata, más que antaño, de desarrollar una democracia social a través la consulta de diversos agentes internos y externos. En lo que concierne a los usuarios, se trata, más que antaño, de formar ciudadanos capaces de ocupar su espacio en una sociedad democrática que ellos mismos contribuyen a hacer surgir.

\subsection{Relación con la dirección de la institución escolar}

Que la participación haya sido decidida de manera más formal o que se haya desarrollado de manera más emergente en la institución social o educativa, de todas maneras no será pertinente ni eficaz sino se desarrolla en el marco de prioridades claras tanto en el pilotaje como en la coordinación.

Por otra parte, está claro que participar en todo momento y en todos los niveles no tiene pertinencia salvo que se trate de pequeñas instituciones o de pequeños equipos creativos, en momentos específicos.

Dicho de otra manera, se trata de plantear, al menos en el comienzo y en el final de una acción, las preguntas clásicas:

- ¿Para quién participamos? ¿Por qué? y ¿Para qué?

- ¿En qué momento? ¿Con qué frecuencia? ¿De qué manera?

- ¿Con qué grado de decisión participamos (información, consulta, decisión encuadrada, codecisión, decisión autónoma)?

- ¿En qué nivel de decisión (estratégica, organizacional, operacional)?

Las respuestas serán diferentes según la filosofía de las personas, según el grado de autonomía y de convicción de éstas. Ellas también diferirán según el tipo de producción o de servicio de la institución. Finalmente, diferirán según las obligaciones del medio y del grado de urgencia, así también según la cultura del país y de la institución en ella misma.

\footnotetext{
${ }^{10}$ in CJEF, Passons l'éponge. Propositions pour recréer l'école, Bruselas: 1994, p. 8.
} 


\subsection{La administración y la gestión participativas entendidas como proceso}

Colaboraciones y participaciones no se crean en un día sobre un terreno no sensibilizado. A este respecto, el ejemplo de la dificultad de instalar gestiones participativas, tanto en el mundo económico como en el político, en países que emergen de una dictadura es elocuente.

El desarrollo de una cultura de participación necesita un conjunto de herramientas, de medios y de modos de comportamiento. La apropiación de estos elementos es progresiva. Esta apropiación necesita una educación, una pedagogía, de manera tal que permita a cada uno vivir activamente su existencia de ciudadano.

Una cultura de participación se construye progresivamente sobre la base de pequeñas acciones logradas, de confianzas conseguidas poco a poco, del desarrollo de competencias de escucha, del análisis y de la expresión. Así por ejemplo, llevar a los padres en la escuela a una situación de decisión o de defensa de un proyecto común, conducir a los ciudadanos a participar de una acción municipal, no podrá realizarse a menos que, previamente, los agentes hayan tenido la ocasión de conocerse, de sentirse en confianza los unos con los otros, de intercambiar francamente y de cuestionarse escuchando a los otros. Dicha cultura de participación no se mantendrá a menos que, posteriormente, la puesta en marcha, la evaluación y los reajustes se realicen en el mismo espíritu. Tenemos en mente, en lo que concierne la puesta en marcha de consejos de participación en las escuelas hace algunos años, numerosos ejemplos negativos de jóvenes y padres decepcionados.

Estas reflexiones no son nuevas. Me referiré aquí con agrado, a lo que, en los años ochenta, Jacky Beillerot llamaba "movimientos en las instituciones" (pedagogía institucional, sociopsicoanálisis en el dominio educativo, social o médico, dispositivos de expresión colectiva para los alumnos...) En dichos casos no se trataba de movimientos sociales voluntaristas, culturales e ideológicos, no se trataba tampoco de reivindicaciones únicamente económicas, ni de reivindicaciones que atañían a la vida privada, sino de la búsqueda de nuevas maneras de organizarse, de asociarse y de cooperar en el trabajo y la organización de éste $\square$ Uno se da cuenta cuánto, para dar al individuo y a las colectividades más poder "sobre" sus actos (autonomía de la acción con relación a lo que ellos tienen que realizar) que

\footnotetext{
${ }^{11}$ Jacky Beillerot, in Sociopsychanalyse, pratiques d'un pouvoir plus collectif aujourd'hui, París: PBP, p. 7.
} 
poder "de" sus actos (control del seguimiento y de las consecuencias posteriores de lo que ellos realizan), una gestión progresiva de análisis y de debate fundada en apuestas reales, es la única manera de sobrepasar la desmovilización y el desinterés, y de reconstruir el placer de la acción organizada.

Las corrientes organizacionales de la empresa actual, tanto en él ámbito mercantil como no mercantil, rechazan porfiadamente los términos de empowerment (ponerse en situación de poder, de control, de responsabilización en su acción) conjugado al accountability (imputabilidad, rendición de cuentas). Aunque se trate de otro ángulo de acercamiento fundado en la búsqueda de eficacia, se subraya la misma necesidad de poder sobre su acto y la misma necesidad de autonomía de los actores.

\section{$3 \quad$ Lugar de la participación en las nuevas regulaciones sociales y educativas de hoy en día[1]}

Actualmente, en el conjunto de países occidentales se observan las mismas presiones de contexto que influencian la evolución de las instituciones educativas y sociales. Retomemos de algunos autores, la puesta en evidencia de algunas de sus características. No es obvio (podríamos referirnos aquí a los estudios de Cerisis en Charleroi, Bélgica, sobre las representaciones de los educadores) que los operadores hayan integrado estos nuevos elementos profesionales, incluso si aquellos que deciden políticamente, los poderes organizadores o los expertos exteriores (consultores) tienden a expresarse en ese sentido.

Puede ser interesante preguntarse sobre las posibilidades emancipadoras de dichas influencias actuales. Encontraríamos aquí también herramientas concretas de investigación. Aqui esta una sintesis de estas tendencias:

A. Evolución de una predominancia de la oferta a una predominancia de la demanda: ello implica nuevas relaciones de influencia (reencuentros, negociaciones) entre usuarios, actores y colaboradores externos.

\footnotetext{
${ }^{12}$ En este párrafo nos referimos particularmente a Claude Lessard, pp. 91-116, dans Recherche et formation, París: INRP, 35, 2000.
} 
Nos parece que esta situación no facilita especialmente la emancipación social, dado que los comportamientos consumidores solicitados no son necesariamente conducidos a analizar las situaciones en términos de equidad, ni a implicarse en la mejora de éstas.

B. Evolución de un control burocrático y de sus ritualismos a una "petición de cuentas" profesionalizante, que toma en cuenta la complejidad, la singularidad, el contexto y la responsabilidad social. Ello no puede ser realizado sino a través de una concertación profesional local, tanto en razón de la complejidad de la tarea como de la necesidad de coherencia de funcionamiento del conjunto de la organización.

La petición social de cuentas no está necesariamente sostenida por una mirada democrática, ella puede conducir a una búsqueda de puro elitismo y de selección.

C. Una prioridad a la obligación de resultados, en el plano cuantitativo como en el cualitativo: Clarificación de perfiles, elaboración de listas de competencias específicas y transversales, explicitación del servicio, de la evaluación, atención a la equidad. Igualmente, ello necesita informaciones, consultas regulares, estructuradas y concertación entre profesionales.

Nos parece que un trabajo de investigación de equidad en nuestras instituciones complejas no puede dispensarse de un pilotaje de elementos objetivos de apreciación. Considerando esto, la obligación de resultados hacia el desarrollo de competencias puede transformarse en una preocupación democrática. Incluso, hay que ver como han sido descritas y negociadas dichas competencias y vigilar a que las variables de contexto sean tomadas en cuenta en el análisis...

D. Evolución de un sistema central controlado a unidades participativas, fundadas en un proyecto educativo construido localmente, y en el cual el usuario tiene un lugar importante. Ello modifica los juegos de influencias y las colaboraciones, conduciendo en principio a las partes constituyentes hacia codecisiones estratégicas.

Estas colaboraciones, si conducen a una mayor participación de los actores y al desarrollo de éstos (si las condiciones de confianza y de formación se hayan presentado), no conducen automáticamente a un progreso democrático generalizado y no protegen del peligro de la dualización. 
E. Evolución de servicios "prêts-à-porter", realizado por profesionales aislados, a una recentración en el aprendizaje y la organización que aprende.

Esta característica nos parece una condición necesaria para la construcción de una vida en común que sea democrática. En lo que concierne a aquellos que aprenden por ejemplo, la participación primera es sin duda, aquella de la instalación de dispositivos de trabajo en común, reconociendo la heterogeneidad y la diversidad, el desarrollo de competencias de escucha y de cooperación, de expresión de sus argumentos, de exteriorización de sus criterios sometidos a la opinión de sus vecinos, de compartir las alternativas para desarrollar en conjunto caminos de aprendizaje. En lo que concierne a los formadores, esto se reencuentra con la construcción de nuevas prácticas (trabajo por competencias, por ciclos, por proyectos). Estos modos de funcionamiento necesitan un nuevo sitio del encuadre y del acompañamiento hacia una autorregulación colectiva y compartida, basada en la aceptación de cambios, de transformaciones, en el ejercicio de un vivir social conjunto solidario, sobrepasando la búsqueda de competición y de logro personal.

4 Condiciones para una participación eficaz y durable al servicio de la emancipación social.

Una participación no piloteada avanza hacia disfuncionamientos, conflictos y a la pérdida de confianza en sus efectos. Así, el mejor decreto sobre la concertación no tendrá efecto por sí mismo si no está acompañado de dispositivos de información, de incitación y de sostén.

¿Qué debe incluir una participación para ser eficaz?

En primer lugar, debemos hacer frente a una participación real, a apuestas reales que correspondan a una influencia real sobre la acción en mira, si se desea que los colaboradores se impliquen. Citemos aquí el ejemplo de los profesores que son informados acerca de la construcción de un nuevo edificio escolar y que, una vez construido, no demuestran deseos de apropiárselo (esta información constituía un acto simbólico importante, pero desvitalizado por la ausencia de concertación entre dirigentes, arquitectos y usuarios). O, por ejemplo, aquellos alumnos y aquellos padres, teniendo la oportunidad de participar a un "Consejo de participación", habiendo aportado esfuerzos y energía para 
contribuir à la reflexión, poniéndose desmotivados después algunas semanas luego de que no se hayan tomado en cuenta sus aportes.

En lo que concierne a la participación en ella misma, evidenciaremos la importancia:

- de una coordinación del proceso, de manera de no enfrentar resultados contradictorios entre micro-participaciones múltiples (¿qué actor garantizará el entrelazado entre los diferentes niveles?);

- de una metodología, de manera de no encontrar excesos de flujos y de pérdidas de tiempo (¿quién anima, quién decide la animación? ¿se utilizará una lluvia de ideas, la consultación de opiniones una a una, un voto secreto, un Phillips 6,6? ¿Cómo se organizará la memoria del grupo?);

- de un análisis riguroso de las posturas y los objetivos que persiguen los distintos colaboradores, de manera de evitar la exacerbación de los intereses personales;

- de un pilotaje y una puesta en marcha, de manera tal que no se consoliden los disfuncionamientos y de regular la acción;

- de los objetivos y de las orientaciones generales precisados, de manera de no encontrar exceso de incoherencias desde la base a la cúpula (¿Cómo la participación contribuirá a cumplir mejor nuestra misión?).

Las reflexiones presentada más arriba, sin ser desdeñables, son análisis relativos a la ingeniería organizacional, la que, lamentablemente, excluye a menudo al actor de base, sin duda porque no lo toma directamente en cuenta y lo deja desamparados frente a la operacionalización. Cuando los equipos participativos funcionan, podemos observar concretamente en los actores participantes ciertos modos de funcionamiento precisos.

Para explicitar nuestros propósitos, tomemos un análisid 13 del funcionamiento de Consejos de Participación en un conjunto de escuelas secundarias.

\footnotetext{
${ }^{13}$ Eddy De Gelaen et Christian Hanot, L'école démocratique, Louvain-la-Neuve: FOPES, 1999, p. 32. Esta memoria analiza comparativamente, el funcionamiento de cinco consejos de participación en la región de Bruselas - Brabant wallon.
} 
Una primera observación es que la existencia del Consejo de Participación en tanto tal, no implica en nada un funcionamiento democrático. Una segunda observación pone en evidencia que el funcionamiento democrático parece estrechamente relacionado:

- al cuestionamiento del poder por el conjunto de los actores, y la aceptación del conflicto como fuente de producción y de cambio;

- a la estructuración de los actores bajo una forma representativa, globalmente y en cada categoría;

- a la participación de todos en el proceso de decisión (todas las categorías, así como el conjunto de los actores de cada una de ellas);

- al sostén, a la formación y a la motivación de los actores, a través de dispositivos de formación y de análisis de prácticas, y a través de un modo de liderazgo transformador;

- la toma en cuenta de la temporalidad del proyecto y el establecimiento de un plan progresivo y realista de realización.

\section{$5 \quad$ Planteamiento de tensiones para las instituciones que aprenden}

La participación, ya lo hemos dicho, no es ni mágica ni homogénea. Ella entrecruza posturas y actores variados, siempre en cooperación conflictiva, así honestos y competentes que sean éstos últimos. La participación raramente es "dada", ella es muy a menudo un constructo social en un sistema complejo en constante evolución.

En el encuadre sistémico, que es el nuestro, no produciremos una lista de "buenas" o de "malas" participaciones. Pero, lo que podemos hacer es identificar, reconocer las tensiones que atraviesan toda acción realizada, los dilemas que fundan toda acción democrática, las paradojas en los valores, las posiciones, los roles. Es a partir de estos referentes que los debates y la concertación podrán construirse, y que una decisión colectivamente fundada podrá ser tomada, con relación a los valores, a las prioridades, a los acuerdos. Es, lo sabemos bien hoy en día en los estudios relativos al aprendizaje, sólo en el conflicto de las representaciones en donde puede construirse un conocimiento enraizado y comprometido, sea éste individual o colectivo.

Citemos algunos ejemplos de tensiones identificables en el marco de una acción participativa: 
- entre democracia representativa (consejos, delegados) y/o democracia participativa (apertura a proyectos colectivos) de los actores de la comunidad escolar,

- entre justicia distributiva y/o justicia diferenciada,

- entre participación con el exterior y/o con los actores internes,

- entre una transmisión de la herencia y/o de la creación de nuevas dimensiones,

- entre la atención al individuo y/o la atención a la sociedad global,

- entre una dimensión igualitaria y/o una dimensión elitista...

Para ello es necesario que sean analizadas y "administradas" las diferencias entre lo que es buscado y lo que es realizado en la institución, y que los lugares y tiempos sean reservados a dicho trabajo. Es necesario también que sean coordinadas coherentemente las diversas facetas de la "gestión de recursos humanos": reclutamiento y acogida, formación y evaluación, concertación, gestión de proyectos... Si no hay continuidad, un esfuerzo puntual de participación no permanecerá por largo tiempo como legítimo a los ojos de aquellos que constituyen el objeto.

No podemos terminar sin subrayar un punto importante que no siempre es tomado en consideración en las reflexiones sobre la participación. Es el hecho que, si apuntamos hacia una emancipación social, la participación de un conjunto de actores será efectiva sólo si cada uno de dichos conjuntos (estudiantes, profesores, equipo de dirección, padres, educadores...) existe por sí mismo, es reconocido como tal, y dispone de la posibilidad de ejercer un trabajo participativo democrático en el seno de su colectividad. Yo encuentro todavía, de vez en cuando, directores de establecimiento para los cuales los encuentros y discusiones de profesores, de estudiantes, de educadores o de padres, aisladamente, de manera privada, como grupos independientes, es percibido negativamente. Opuestamente a los temores de esos dirigentes, la posibilidad y el aprendizaje de la expresión colectiva por los alumnos (posibilidad de reunirse entre ellos, de poder expresarse y dar su opinión sobre lo que ellos viven en común en su vida escolar) como esta misma posibilidad abierta a otros colaboradores, no puede sino conducir a una mejor comunicación del conjunto del establecimientd 14 Estos dispositivos son puestos en marcha en más de un establecimiento escolar, incluso con niños muy pequeños, considerando, obviamente, que ellos deben ser acompañados de un aprendizaje, a veces difícil, de la participación colectiva y de formas de

${ }^{14}$ Claire Rueff-Escoubès y Jean-François Moreau, La démocratie dans l'école, París: Syros, 1987. 
autorregulación de un grupo, al seno de él mismo así como con respecto a los otros grupos que constituyen el establecimiento. Para ello todavía hay que reconocer a cada una de dichas colectividades la posibilidad de ejercer un poder y una palabra, y que se le consagren los recursos necesarios. Una toma de posición de ese tipo se conjuga, evidentemente, con una posición democrática comprometida. Si dicha convicción no está presente, la participación se enfrentará a las más grandes dificultades.

\section{Conclusión}

En conclusión, insistiremos en el importante trabajo de continuidad y de coherencia necesaria al seno de las instituciones. Todas las facetas de la vida de ésta deben, tanto como pueda hacerse, referirse a convenciones claras, fundadas en el respecto y el reconocimiento de todas las personas y en concordancia de unos y otros. La participación no puede ser generalizada y activada en permanencia, las rutinas y las estandarizaciones son necesarias igualmente, las acciones y búsquedas individuales pueden imponerse en este marco. Ello no tiene nada de contradictorio con el desarrollo de una cultura de la participación y instalación de un tiempo y de lugares de trabajo en común.

Es decir, que es tarea de los responsables de garantizar al "vivir comunitario" un sostén y un apoyo, tanto en la construcción de las decisiones y de normas, como en la aplicación y el seguimiento de éstas. Es decir, la necesidad de dar, a aquellos que no las disponen, herramientas emancipadoras de descodificación y de palabra. Es decir, el sitio importante del liderazgo y del encuadre de proximidad a todas las etapas de las acciones participativas, sabiendo que el aprendizaje del "vivir comunitario", permanente y progresivo no está nunca terminado. 


\section{Referencias}

Beillerot Jacky, in Sociopsychanalyse, pratiques d'un pouvoir plus collectif aujourd'hui. París: PBP.

CJEF. (1994). Passons l'éponge. Propositions pour recréer l'école. Bruselas.

Crozier Michel. (1977). L'acteur et le système. Paris: Seuil.

Hermel Philippe. (1988). Le management participatif. París: Les éditions d'organisation.

Lessard Claude. (2000). Recherche et formation. París: INRP, 35.

Mothe-Gautrat Daniel. (1984). Méthode d'évaluation des systèmes participatifs. París: CNRS.

Peeters Luc. (1991). La concertation dans l'enseignement. Bruselas: De Boeck.

Senge Peter. (1990). The Fifth Discipline. Doubleday. 